\title{
Vestibular Rehabilitation Therapy for Management of Concussion: A Critically Appraised Topic
}

\author{
Matthew Booth, ATC,,$^{1,2}$ Jacob Powell, ATC, ${ }^{3}$ Patrick O. McKeon, PhD, ATC, CSCS, ${ }^{1}$ \\ and Jennifer M. Medina McKeon, PhD, ATC, CSCS $^{1}$ \\ ${ }^{1}$ Ithaca College; ${ }^{2}$ University of North Carolina at Chapel Hill; ${ }^{3}$ Temple University
}

\begin{abstract}
Focused Clinical Question: In patients who have sustained a concussion, does vestibular rehabilitation therapy improve clinical symptoms of dizziness and self-reported balance deficits and/or influence return-to-participation timelines beyond that of preintervention scores or standard care? Clinical Bottom Line: The evidence supported the use of VRT to attenuate clinical symptoms of dizziness and self-reported balance deficits.
\end{abstract}

Keywords: injury management, traumatic brain injury, postural control

\section{Clinical Scenario}

Sport-related concussion is a complex pathophysiological process, resulting in acute and, at times, persistent, functional somatic, cognitive, and affective signs and symptoms. ${ }^{1}$ Two of the most common clinical problems following a concussion are dizziness and poor balance

\section{Key Points}

Vestibular rehabilitation therapy (VRT) appears to have a moderateto-strong effect on reducing symptoms of dizziness and balance deficits in patients with concussions.

Recognition of vestibular symptoms in a concussed patient is strongly recommended, and an active, symptom-based VRT approach is supported by the research evidence.

In certain professions, such as athletic training, where education in VRT is currently not standard, an interprofessional approach to treating concussed athletes is strongly recommended. control. ${ }^{2}$ Persistent dizziness and balance deficits lengthen return-to-play (RTP) timelines, ${ }^{3}$ which can be frustrating for athletes and other stakeholders, and significantly impact activity and social participation in daily life. While there are many possible root causes for dizziness and balance deficits subsequent to a concussion, dysfunction within the vestibular system may be a key contributing factor. ${ }^{2}$

Vestibular rehabilitation therapy (VRT) is a rehabilitation paradigm for treating vestibular disorders marked by increased dizziness, poor gaze stabilization, and loss of balance. ${ }^{4}$ VRT for patients with vestibular disorders often includes treatment techniques such as gaze stabilization, as well as task manipulation in static (e.g., standing balance with double- or single-limb stances, use of foam pads, eyes open/closed) and dynamic (e.g., walking with fixed gaze, obstacle avoidance, or

Booth, McKeon, and Medina McKeon are with Exercise \& Sport Sciences, Ithaca College, Ithaca, NY. Booth is also with University of North Carolina at Chapel Hill, Chapel Hill, NC. Powell is with the Department of Kinesiology, Temple University, Philadelphia, PA. Medina McKeon (jmckeon@ithaca.edu) is corresponding author. head movements) balance drills. ${ }^{5}$ These treatment techniques are commonly performed by a physical therapist or neurorehabilitation specialist. ${ }^{4}$ Both subjective patient reports and objective tests of balance or gaze stability are used as outcomes to demonstrate improvement. ${ }^{4}$

Recently, greater awareness has been raised concerning the necessity to utilize an active, symptom-based intervention approach to concussion management, such as subsymptom threshold graded exercise, cognitivebehavioral therapy, and academic accommodations, in conjunction with rest. ${ }^{6}$ VRT has also been recommended as an emerging series of treatment strategies for resolving vestibular symptoms in those with concussion. ${ }^{7-9}$ Despite a high incidence of dizziness and balance deficits reported after concussion, the evidence for VRT effectiveness as a means to resolve these concussion symptoms is relatively sparse. ${ }^{9}$

\section{Focused Clinical Question}

In patients who have sustained a concussion [P], does VRT [I] improve clinical symptoms of dizziness and selfreported balance deficits and/or influence RTP timelines [O] beyond that of preintervention scores or standard care $[\mathrm{C}]$ ?

\section{Search Strategy}

\section{Data Sources}

A computerized search of databases (PubMed and EBSCOHost (SPORTDiscus, MEDLINE, CINAHL) was completed in September 2018 regarding vestibular rehabilitation and the management of concussion. Figure 1 displays the search term phrases and combinations, and search results. Studies were limited to those published in English after 2010. Reference sections of retrieved studies were hand searched. 
PUBMED Search

\begin{tabular}{|c|l|c|}
\hline Step & \multicolumn{1}{|c|}{ Terms } & $\begin{array}{c}\text { Studies } \\
\text { Identified }\end{array}$ \\
\hline $\mathbf{1}$ & concuss $^{*}$ vestibul $^{*}$ rehab $^{*}$ & $\mathrm{~N}=53$ \\
\hline $\mathbf{2}$ & Step 1 + AND dizzi & $\mathrm{N}=21$ \\
\hline $\mathbf{3}$ & Step 2 + NOT review & $\mathrm{N}=13$ \\
\hline
\end{tabular}

EBSCOHost Search (CIHNAL, MEDLINE, SPORTDiscus, Military \& Government Collection, Health Source)

\begin{tabular}{|c|l|c|}
\hline Step & \multicolumn{1}{|c|}{ Terms } & $\begin{array}{c}\text { Studies } \\
\text { Identified }\end{array}$ \\
\hline $\mathbf{1}$ & vestibul $^{\star}$ AND (treatment or rehabilitation or physical therapy) AND concuss ${ }^{*}$ in sports NOT combat & $\mathrm{N}=46$ \\
\hline $\mathbf{2}$ & Step 1 + NOT review & $\mathrm{N}=19$ \\
\hline $\mathbf{3}$ & Step 2 + NOT diagnosis & $\mathrm{N}=12$ \\
\hline
\end{tabular}

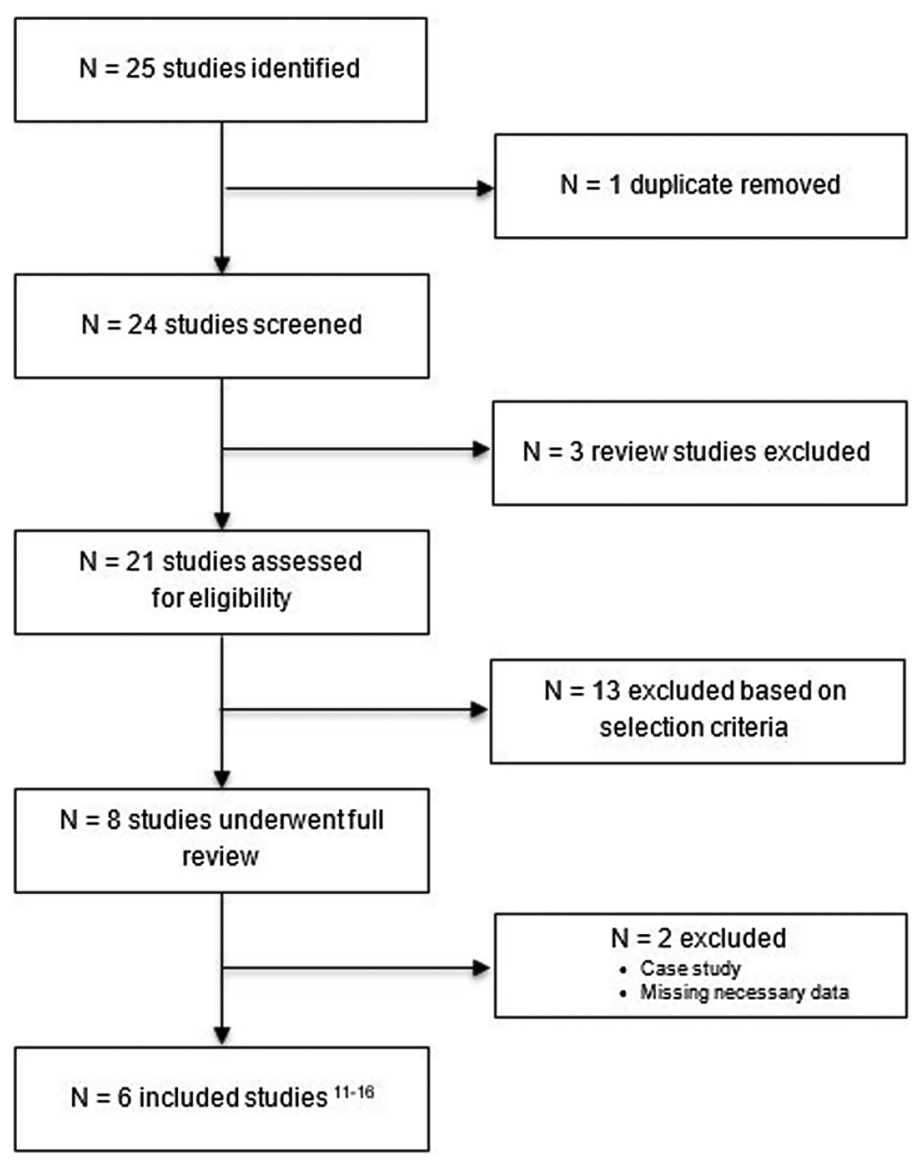

Figure 1 - Summary of search strategy and results.

\section{Study Selection}

Criteria for selection required that original studies: (a) included participants who sustained a concussion and were experiencing unresolved vestibular symptoms or deficits; (b) were clinical trials or case series; (c) utilized VRT to treat concussion-related dizziness or balance deficits associated with concussion; and (d) used patient self-reported outcomes to assess dizziness and balance ability in response to treatment. Studies that (a) were reviews of the literature, (b) did not assess the effects of VRT on dizziness symptoms subsequent to a concussion, or (c) were case studies were excluded.

\section{Evidence Quality Assessment}

\section{Evidence Appraisal}

Two different critical appraisal tools were used to identify potential threats to validity, depending on study design. The Physiotherapy Evidence Database (PEDro) scale (for clinical trials) and the Joanna Briggs Institute (JBI) Critical Appraisal Checklist (for case series) were both used to assess internal validity of the included studies, as appropriate. Two authors (MB \& JM) independently assessed included studies. There were no discrepancies in scores. 
Table 1 Evidence Appraisal of Included Studies

\begin{tabular}{|c|c|c|c|c|c|}
\hline Author & Year & Study Design & OCEBM Level of Evidence & JBI & PEDro \\
\hline Kleffelgaard et al. ${ }^{14}$ & 2019 & $\mathrm{RCT}$ & 2 & N/A & 8 \\
\hline Schneider et al. ${ }^{13}$ & 2014 & RCT & 2 & N/A & 8 \\
\hline Kontos et al. ${ }^{15}$ & 2018 & Case series & 4 & 8 & N/A \\
\hline Moore et al. ${ }^{16}$ & 2016 & Case series & 4 & 9 & N/A \\
\hline Kleffelgaard et al. ${ }^{12}$ & 2016 & Case series & 4 & 8 & N/A \\
\hline Alsalaheen et al. ${ }^{11}$ & 2010 & Case series & 4 & 8 & N/A \\
\hline
\end{tabular}

Abbreviations: JBI = Joanna Briggs Institute Critical Appraisal Checklist for Case Series; OCEBM = Oxford Center for Evidence-Based Medicine; PEDro = Physiotherapy Evidence Database scale for quality assessment of randomized clinical trials; RCT = randomized clinical trial.

\section{Data Extraction}

We sought to evaluate the effectiveness of VRT related to patientoriented outcomes of self-reported dizziness and balance improvements as well as return-to-sport participation. The selected outcomes of interest extracted, when available, were:

1. Dizziness, as measured by the Dizziness Handicap Inventory (DHI) (25 items, 4 points each, 100 points is "worst symptoms")

2. Dizziness, as measured by the Numeric Pain Rating Scale for dizziness (NPRS-dizziness) (11 items, 1 point each, 10 points is "worst symptoms")

3. Dizziness, as measured by the Vertigo Symptom Scale for vertigo-balance symptoms (VSSv) (8 items, 4 points each, 32 points is "worst symptoms")

4. Self-reported balance ability, as measured by the ActivitiesSpecific Balance Confidence scale (ABC) (100 points, 100 points is "best confidence in balance")

\section{Return-to-Participation (RTP)}

Means and standard deviations were extracted for continuous data for the DHI, NPRS-dizziness, VSSv, and ABC. Further, dichotomous data were extracted for RTP (return, no return), and for complete recovery from dizziness symptoms for the DHI (no dizziness, dizziness), NPRS-dizziness (dizziness, no dizziness), and the $\mathrm{ABC}$ (perceived balance problems, no perceived balance problems). Relevant sample sizes from each study were also extracted. Tables 2 (randomized clinical trials [RCTs]) and 3 (case series), presented later, contain the characteristics of the included studies.

\section{Summary Measures}

Data regarding the effects of VRT on postconcussion symptoms were analyzed utilizing two methods. For continuous data, Hedges' $\mathrm{g}$ effect sizes (ES) [95\% CIs] were calculated to determine the preto-posttreatment effects on individuals who underwent VRT. For dichotomous data, risk ratios (RR) [95\% CIs], relative risk increase (RRI), and numbers-needed-to-treat to benefit (NNTB) were calculated for the effect of VRT versus standard care for clearance for RTP, absence of dizziness (as measured by the DHI and NPRSdizziness), and full balance confidence (as measured by the ABC). Beyond interpreting the magnitude of the ES and the confidence intervals based on the respective critical value ( 0 for Hedges' $\mathrm{g}, 1$ for RR, and infinity for NNTB), the credible lower limit (CLL $95 \%$ ) was also considered. The CLL $95 \%$ is defined as the smallest effect that corresponds to the accuracy of the effect estimate within a given population and is defined as the lower limit of the $95 \%$ confidence interval. ${ }^{10}$ This estimate represents the "worst-case scenario" for the application of a particular treatment.

\section{Results of Search}

\section{Summary of Search and Quality Assessment}

Six studies met selection criteria. ${ }^{11-16}$ Study design varied, with two RCTs ${ }^{13,14}$ and four case series ${ }^{11,12,15,16}$ meeting eligibility requirements. Both studies assessed with PEDro 13,14 scored $8 / 10$. For the four studies assessed with the JBI, ${ }^{11,12,15,16}$ scores ranged from 8 to 9 . Table 1 is a summary of the evidence appraisal. Patients in the included studies consisted of both children and adults. Time since injury ranged from less than 1 week to over 3 years. Study characteristics, including patient demographics, time since injury time frames, and baseline scores on selected outcomes located in Tables 2 (RCTs) and 3 (case series).

\section{Best Evidence}

Pre-Post VRT. For patients who underwent VRT, there was an improvement in symptoms after treatment compared to baseline (Figure 2). The effect of this treatment for the reduction of dizziness (DHI and VSSv) and improved perception of balance (ABC) was strong (pooled ES $=-1.5[-2.4,-0.5], p<.002$ ). The $\mathrm{CLL}_{95 \%}$ of the pooled effect indicated that, in the population, a moderate effect would be anticipated in balance confidence and dizziness resolution.

VRT vs. Standard Care. Compared to patients who were treated with standard care alone, there was a substantial increase in the chances of complete symptom recovery and for RTP. There was also a substantial chance of complete recovery, as measured by clearance for RTP and, also, for scoring a " 0 dizziness score" on the DHI, with confidence intervals that did not encompass 1 (Figure 3). On the NPRS and the ABC, VRT still increased the chance of complete recovery, however CIs did encompass 1 (Figure 3). When considering the CLL $95 \%$ for these outcomes, the DHI and ABC were relatively close to 1 , indicating that the worst outcome for those who received VRT was similar to standard care. The CLL $95 \%$ of the NPRS indicated that VRT may have resulted in increased reported dizziness for some patients when compared to standard care.

NNTBs for complete recovery on all outcomes were small, meaning very few patients would need to be treated to have a desired benefit. It was estimated that between two to four individuals would need to be treated with VRT to obtain complete recovery from dizziness symptoms, self-reported balance improvement, and ability to RTP in one patient, depending on the outcome 
Table 2 Characteristics of Included Studies (OCEBM Level 2)

\begin{tabular}{|c|c|c|c|c|}
\hline \multirow[b]{2}{*}{ Authors } & Patients & \multirow[b]{2}{*}{ Control Group (Range) } & \multirow[b]{2}{*}{ VRT Protocol } & \multirow[b]{2}{*}{ Outcomes } \\
\hline & Experimental Group (Range) & & & \\
\hline \multirow{6}{*}{$\begin{array}{l}\text { Kleffelgaard } \\
\text { et al. }{ }^{14}\end{array}$} & males $=10 ;$ females $=23$ & males $=9 ;$ females $=22$ & \multirow{6}{*}{$\begin{array}{l}\text { 8-week intervention, group appoint- } \\
\text { ments with physical therapist } \\
\text { Both groups: VRT was customized to } \\
\text { address impairments and functional } \\
\text { limitations in oculomotor function, gait, } \\
\text { and balance } \\
\text { Experimental group: VRT + psycho- } \\
\text { logical treatment addressed self- } \\
\text { efficacy, coping with symptom pressure } \\
\text { and disease burden; HEP included, with } \\
\text { exercise diary }\end{array}$} & \multirow[t]{6}{*}{ DHI, VSSv } \\
\hline & $\overline{\mathrm{x}}(\mathrm{SD})$ age $=37.6(12.3)$ years & $\overline{\mathrm{x}}(\mathrm{SD})$ age $=41.2(13.6) \mathrm{yrs}$ & & \\
\hline & $\begin{array}{l}\text { MOI: MVA }(\times 6) \text {, violence }(\times 2) \\
\text { sport \& other }(\times 6) \text {, fall }(\times 19)\end{array}$ & $\begin{array}{l}\text { MOI: MVA }(\times 4) \text {, violence } \\
(\times 4) \text { sport \& other }(\times 1) \text {, } \\
\text { fall }(\times 22)\end{array}$ & & \\
\hline & $\begin{array}{l}\bar{x}(\mathrm{SD}) \text { time since injury }= \\
4.0(2) \text { months }\end{array}$ & $\begin{array}{l}\overline{\mathrm{x}}(\mathrm{SD}) \text { time since injury }= \\
3.5 \text { (2) months }\end{array}$ & & \\
\hline & $\overline{\mathrm{x}}(\mathrm{SD})$ baseline $\mathrm{DHI}=47.9$ (16.6) & $\begin{array}{l}\bar{x}(\mathrm{SD}) \text { baseline } \\
\mathrm{DHI}=41.4(19.2)\end{array}$ & & \\
\hline & $\overline{\mathrm{x}}(\mathrm{SD})$ baseline $\mathrm{VSSv}=10.9 \pm 6.0$ & $\begin{array}{l}\bar{x}(S D) \text { baseline } \\
\mathrm{VSSv}=10.2 \pm 6.6\end{array}$ & & \\
\hline \multirow{7}{*}{$\begin{array}{l}\text { Schneider } \\
\text { et al. }{ }^{13}\end{array}$} & males $=11 ;$ females $=4$ & males $=7 ;$ females $=9$ & \multirow{7}{*}{$\begin{array}{l}\text { 8-week intervention for both groups, } \\
\text { weekly appointments with } \\
\text { physiotherapist } \\
\text { Both groups: Treatment included ROM } \\
\text { exercises, stretching, postural reeduca- } \\
\text { tion, rest, and HEP included with } \\
\text { exercise diary } \\
\text { Experimental group: VRT included a } \\
\text { combination of c-spine rehabilitation } \\
\text { (joint mobilization, therapeutic exer- } \\
\text { cises for retraining craniovertebral } \\
\text { flexors and extensors, sensorimotor } \\
\text { retraining) and vestibular rehabilitation } \\
\text { (gaze stabilization, adaptation exercise, } \\
\text { standing balance maneuvers) }\end{array}$} & \multirow{7}{*}{$\begin{array}{l}\text { ABC, DHI, } \\
\text { NPRS-dizziness } \\
\text { RTP }\end{array}$} \\
\hline & $\overline{\mathrm{x}}$ age $=15$ (range $=12$ to 27 ) years & $\begin{array}{l}\bar{x} \text { age }=15 \text { (range }=13 \text { to } \\
30 \text { ) years }\end{array}$ & & \\
\hline & MOI: sport $(\times 15)$ & MOI: sport $(\times 16)$ & & \\
\hline & $\begin{array}{l}\text { Median (range) time since injury = } \\
1.7(0.25 \text { to } 9) \text { months }\end{array}$ & $\begin{array}{l}\text { Median (range) time since } \\
\text { injury }=1.5 \text { (1 to } 4.5) \\
\text { months }\end{array}$ & & \\
\hline & $\begin{array}{l}\text { Median (range) baseline } \\
\mathrm{ABC}=80(40 \text { to } 95)\end{array}$ & $\begin{array}{l}\text { Median (range) baseline } \\
\mathrm{ABC}=85(40 \text { to } 100)\end{array}$ & & \\
\hline & $\begin{array}{l}\text { Median (range) baseline } \\
\mathrm{DHI}=46(6 \text { to } 84)\end{array}$ & $\begin{array}{l}\text { Median (range) baseline } \\
\text { DHI }=42(0 \text { to } 66)\end{array}$ & & \\
\hline & $\begin{array}{l}\text { Median (range) NPRS- } \\
\text { dizziness }=3(0-8)\end{array}$ & $\begin{array}{l}\text { Median (range) NPRS- } \\
\text { dizziness }=5(0-8)\end{array}$ & & \\
\hline
\end{tabular}

Abbreviations: $\mathrm{ABC}=$ Activities-Specific Balance Scale (0-100 worst-best); DHI = Dizziness Handicap Inventory $(0-100$ best-worst $)$; HEP = home exercise program; MOI = mechanism of injury; MVA = motor vehicle accident; NPRS-dizziness = Numerical Pain Rating Scale for dizziness $(0-10$ best - worst $)$; OCEBM = Oxford Centre for Evidence-Based Medicine; ROM = range of motion; RTP = return-to-play; VRT = vestibular rehabilitation therapy; VSSv = Vertigo Symptom Scale for vertigobalance symptoms (0-32 best-worst).

used (Figure 4). The CLL $95 \%$ for the NNTB estimates indicated consistently small numbers to benefit except for the ABC (10 NNTHarm). There is a potential for VRT to have a negative impact on self-reported balance ability.

\section{Clinical Bottom Line}

The evidence supported the use of VRT to attenuate clinical symptoms of dizziness and self-reported balance deficits. However this comes from only six studies, some with limited quality. Patients treated with VRT were $\sim 2 \times$ more likely to have complete recovery from dizziness symptoms. Based on the CLL $\mathrm{CL}_{95}$, for the majority of the outcomes, there may be no benefit from VRT for some patients. As well, there may be key factors that may better predict success of VRT in concussed patients, but these factors remain unknown. Across the majority of outcomes, approximately two to four patients would need to be treated with VRT to completely dissipate vestibular symptoms in one patient. Further, individuals who experienced a complete reduction in vestibular symptoms, following VRT, were 8.5 times more likely to return to sports participation compared to those who received standard care. The CLL $95 \%$ for this estimate indicated that these patients would be at least three times more likely to return to participation. Although some $\mathrm{CLL}_{95 \%}$ did approach the value of equality, these relatively wide CIs were most likely influenced by the small sample size ( $\mathrm{n}=28$ total patients enrolled), thus inflating the variability of results.

\section{Implications for Practice, Education, and Future Research}

The evidence appraised in this Critically Appraised Topic (CAT) supported the use of VRT to attenuate clinical symptoms associated with dizziness and balance deficits compared with standard care. For the outcomes assessed in the present study, VRT appeared to have the strongest effect on reported dizziness, and a moderate effect on self-reported balance ability. Based on the NNTB, it appears that VRT is a promising strategy for enhancing return to participation, especially in sport. ${ }^{13}$

Further, while only assessed in a single study, there was a considerable increased chance of RTP, as a marker of complete recovery from symptoms, in concussed athletes who underwent VRT. For athletes, RTP is often a critical outcome, and under current guidelines, RTP should not take place until the athlete is asymptomatic. ${ }^{1,3}$ Recognition of vestibular dysfunction, patient education on the importance of seeking treatment in VRT, and referral to clinicians who specialize in VRT is paramount. An interprofessional approach that includes the AT, the physician, and the VRT specialist is key to proper diagnosis and treatment for the patient with vestibular symptoms.

The estimates of benefit in this CAT included Hedges' $g$ effect sizes, the risk ratio, and NNTB. Based on the $\mathrm{CLL}_{95 \%}$, the worstcase scenario appears to be no benefit in some patients. There appears to be little chance of potentially making the patients worse 
Table 3 Characteristics of Included Studies (OCEBM Level 4)

\begin{tabular}{|c|c|c|c|}
\hline Author & Patients & VRT Protocol & Outcomes \\
\hline \multirow[t]{3}{*}{ Kontos et al. ${ }^{15}$} & $\begin{array}{l}\text { males }=20 ; \mathrm{n} \text { females }=6 \\
\bar{x}(\mathrm{SD}) \text { age }=37.0(9.6) \text { years } \\
{[\text { range }=22 \text { to } 59]}\end{array}$ & $\begin{array}{l}\text { Individual sessions with rehabilitation specialists } \\
\text { from the clinical research team via phone- and } \\
\text { tablet-based program }\end{array}$ & \multirow[t]{3}{*}{$\mathrm{ABC}, \mathrm{DHI}$} \\
\hline & $\begin{array}{l}\text { MOI: blast }(\times 11) \text {, BFT }(\times 11) \text {, mixed }(\times 4) \\
\text { Time since injury }=\text { at least } 6 \text { months } \\
\text { postinjury }\end{array}$ & $\begin{array}{l}\text { Customized to address impairments and functional } \\
\text { limitations in oculomotor function, gait, and balance }\end{array}$ & \\
\hline & $\begin{array}{l}\bar{x}(\mathrm{SD}) \text { baseline } \mathrm{ABC}=24.0(11.2) \\
\overline{\mathrm{x}}(\mathrm{SD}) \text { baseline } \mathrm{DHI}=52.4(34.0)\end{array}$ & $\begin{array}{l}\text { Interventions included behavioral, vestibular, } \\
\text { visual, and exertional therapy }\end{array}$ & \\
\hline \multirow[t]{3}{*}{ Moore et al. ${ }^{16}$} & $\begin{array}{l}4 \text { males; ages }=18,27,33,37 \text { years } \\
4 \text { females; ages }=22,27,41,44 \text { years }\end{array}$ & $\begin{array}{l}\text { Individual sessions with VRT rehabilitation } \\
\text { specialists }\end{array}$ & \multirow[t]{3}{*}{$\mathrm{ABC}, \mathrm{DHI}$} \\
\hline & $\begin{array}{l}\text { MOI: MVA }(\times 3), \text { sport }(\times 3), \text { fall }(\times 2) \\
\bar{x}(\text { range }) \text { time since injury }=11(0.75 \text { to } 32) \\
\text { months }\end{array}$ & $\begin{array}{l}\text { Customized to address impairments and functional } \\
\text { limitations in oculomotor function (static and } \\
\text { dynamic gaze stabilization), gait (ambulation with } \\
\text { alterations in head positioning and motions), and } \\
\text { balance (sensory organization) }\end{array}$ & \\
\hline & $\begin{array}{l}\bar{x} \pm \text { SD baseline ABC range }=71.6 \pm 24.5 \\
\bar{x} \pm \text { SD baseline DHI range }=53.3 \pm 21.2\end{array}$ & $\begin{array}{l}\text { Progressive aerobic exercise program, HEP } \\
\text { included }\end{array}$ & \\
\hline \multirow[t]{3}{*}{ Kleffelgaard et al. ${ }^{12}$} & $\begin{array}{l}2 \text { males; age }=25,34 \text { years } \\
2 \text { females; age }=40,45 \text { years }\end{array}$ & $\begin{array}{l}\text { 8-week intervention, group and individual sessions, } \\
\text { appointments with physical therapist }\end{array}$ & \multirow[t]{3}{*}{ DHI } \\
\hline & $\begin{array}{l}\text { MOI: MVA }(\times 1) \text {, violence }(\times 1) \text {, fall }(\times 2) \\
\text { Range time since injury }=9 \text { to } 30 \text { months }\end{array}$ & $\begin{array}{l}\text { Customized to address impairments and functional } \\
\text { limitations in oculomotor function, gait, and balance }\end{array}$ & \\
\hline & $\overline{\mathrm{x}}(\mathrm{SD})$ baseline $\mathrm{DHI}=56.5(10.6)$ & $\begin{array}{l}\text { VRT included a combination of c-spine rehabilita- } \\
\text { tion (neuromuscular retraining, sensorimotor } \\
\text { retraining) and vestibular rehabilitation (gaze } \\
\text { stabilization, habituation and adaptation exercises, } \\
\text { standing balance maneuvers, sensory integration); } \\
\text { HEP included, with exercise diary }\end{array}$ & \\
\hline \multirow[t]{3}{*}{ Alsalaheen et al. ${ }^{11}$} & $\begin{array}{l}\text { Evaluated with } \mathrm{ABC} \text { scale: } \text { children }=41 ; \\
\text { adults }=27 \\
\text { Evaluated with DHI scale: } \text { children }=42 ; \\
\text { adults }=27\end{array}$ & $\begin{array}{l}\text { Median duration of VRT was } 33 \text { days (range } \\
7-181 \text { days), performed with physical therapist }\end{array}$ & \multirow[t]{3}{*}{$\mathrm{ABC}, \mathrm{DHI}$} \\
\hline & $\begin{array}{l}\text { MOI: not reported } \\
\text { Median (range) time since injury }=1.9(0.25 \\
\text { to 38) months }\end{array}$ & $\begin{array}{l}\text { Customized to address impairments and functional } \\
\text { limitations in oculomotor function, gait, and balance }\end{array}$ & \\
\hline & $\begin{array}{l}\overline{\mathrm{x}}(\mathrm{SD}) \text { baseline } \mathrm{ABC}=64(27) \\
\overline{\mathrm{x}}(\mathrm{SD}) \text { baseline } \mathrm{DHI}=49(21)\end{array}$ & HEP included & \\
\hline
\end{tabular}

Abbreviations: Abbreviations: ABC = Activities-Specific Balance Scale (0-100 worst-best); BFT = blunt force trauma; DHI = Dizziness Handicap Inventory (0-100 bestworst); $\mathrm{HEP}=$ home exercise program; $\mathrm{MOI}=$ mechanism of injury; MVA=motor vehicle accident; OCEBM=Oxford Centre for Evidence-Based Medicine; VRT $=$ vestibular rehabilitation therapy.

compared to either their preintervention scores or compared to standard care. There may be factors that are critical for enhancing the probability of VRT treatment success, but these currently are not known for those with concussion. ${ }^{9}$ It is important to note that there was no standardization for VRT implementation timelines or exercise selection, making it difficult to ascertain the critical key features for defining treatment success.

While we have included information on time since injury for the participants in the included studies, there was such a wide range in (a) mechanism of injury, (b) time since injury (from $<1$ week to $>3$ years), (c) baseline symptom severity, and (d) duration of treatment. A recommendation for the "best" timeframe to initiate VRT is not clear from these studies. As there is currently no consistency in the evidence for when VRT may be most beneficial, it is premature to make this recommendation in the discussion. In truth, this strengthens the recommendation for VRT in those with postconcussive symptoms of dizziness and balance problems. In these highly variable samples, the results indicate a strong effect for VRT with CIs that either are fairly narrow or indicate that no harm is done. If the variability in above factors was too much of a problem, we would actually see diminished strength of the effect of VRT and more variability in the outcomes. A key feature of these results is that VRT appears to have a strong effect regardless of sample modifiers. Across the six included studies, patients received individualized programs based on their symptoms. Based on the findings, while further research is needed to elucidate treatment success factors, the assess-and-treat and progress-reassess approach $^{9,17}$ appears to be most logical to maximize potential benefits at the level of the individual patient.

Based on the results of the present study, administration of the DHI, the NPRS-dizziness, the VSSv, and the ABC to patients with concussions, in order to identify those who may benefit from VRT, is strongly recommended. The DHI and VSSv are self-reported questionnaires specific to dizziness symptoms. ${ }^{18}$ The NPRSdizziness is a variation of a commonly used pain rating scale, in which the patient identifies, along a $10-\mathrm{cm}$ line, ${ }^{19}$ as to how bad dizziness symptoms were at that time. In addition, other clinical domains including visual ${ }^{2}$ (vision and oculomotor), vestibular ${ }^{2}$ 


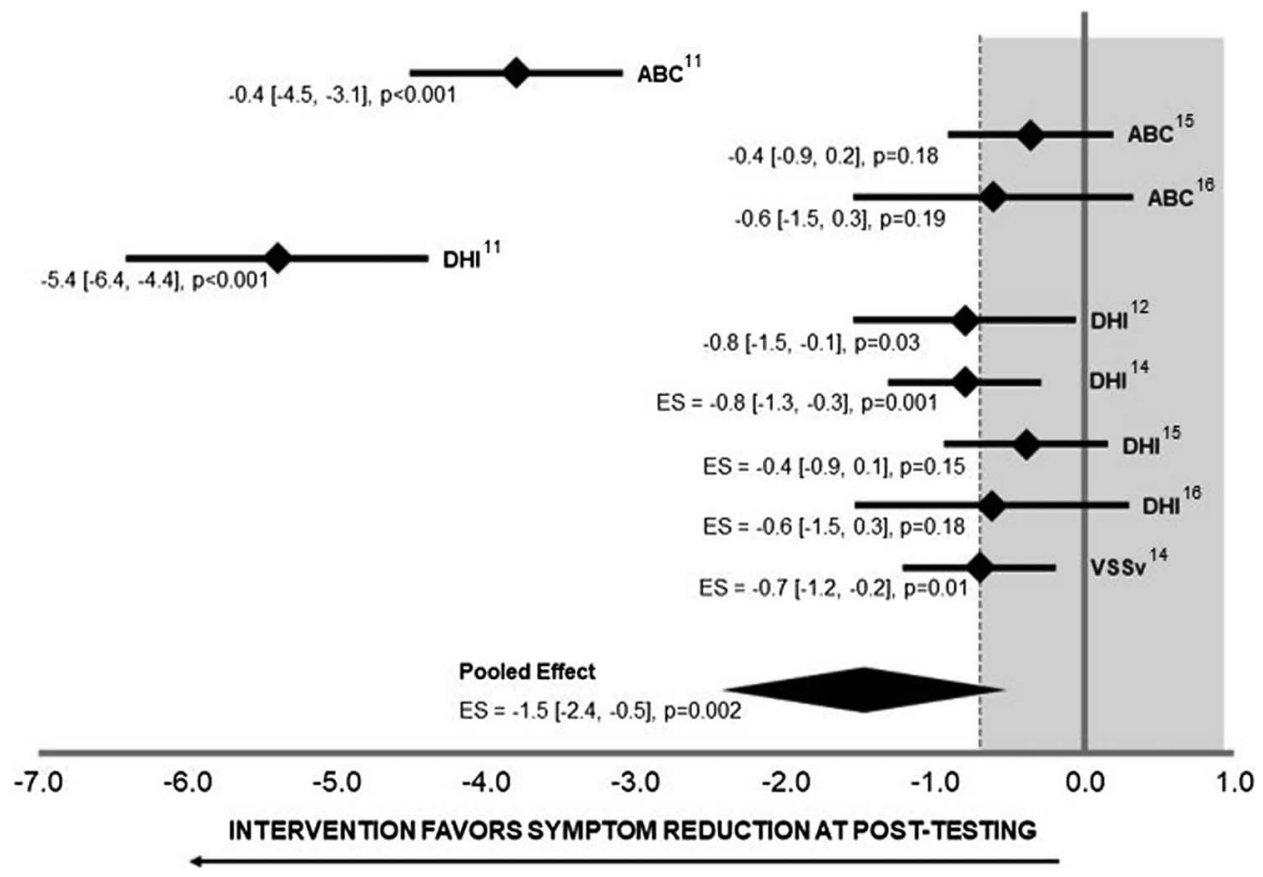

Figure 2 - Magnitude of improvement in balance and dizziness symptoms following 8+ weeks of vestibular rehabilitation (Hedges' g effect sizes with 95\% CIs). There was a strong pooled effect for improvement of balance and dizziness symptoms after treatment with VR, with CIs that did not encompass zero. The dashed gray line indicates the cutoff for a strong effect (Hedges' $g=0.7$ ). Everything to the left, outside of the shaded area, represents a strong effect for the effect of VRT over time in concussed patients. $\mathrm{ABC}=$ Assessment of Balance Confidence scale; $\mathrm{CI}=\mathrm{confidence}$ interval; $\mathrm{DHI}=\mathrm{Dizziness}$ Handicap Inventory scale; ES = effect size; VSSv = Vertigo Symptom Scale for vertigo-balance symptoms.

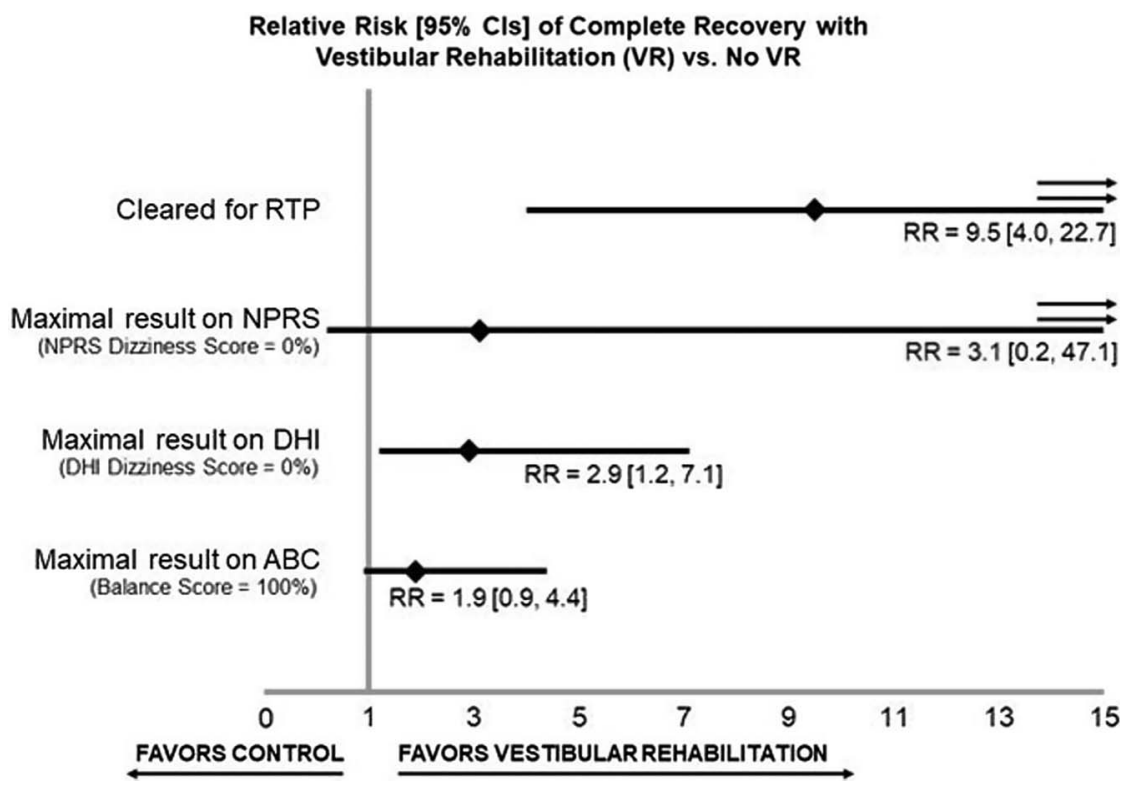

Figure 3 - A depiction of the relative risk [95\% CIs] of RTP, complete recovery of dizziness symptoms, and balance following VR compared to no VR. There was an increased chance of complete recovery with VR, however some CIs did encompass 1 . ABC $=$ Assessment of Balance Confidence scale; $\mathrm{CI}=$ confidence interval; DHI = Dizziness Handicap Inventory scale; NPRS = Numeric Pain Rating Scale for dizziness; RR = relative risk; RTP = returnto-play; $\mathrm{VR}=$ vestibular rehabilitation.

(dizziness and balance), musculoskeletal ${ }^{2}$ (cervical mobility), physiological $^{2,20}$ (exercise intolerance testing), neurocognitive, ${ }^{2}$ and psychological assessment ${ }^{2}$ should be evaluated in patients with prolonged symptoms (symptoms lasting $>10$ days). ${ }^{3}$
Following evaluation, patients exhibiting dizziness symptoms and balance disorders should be educated on the importance of VRT and appropriately referred to a clinician educated in VRT. ${ }^{1}$ The treatment associated with these symptoms and deficits is 


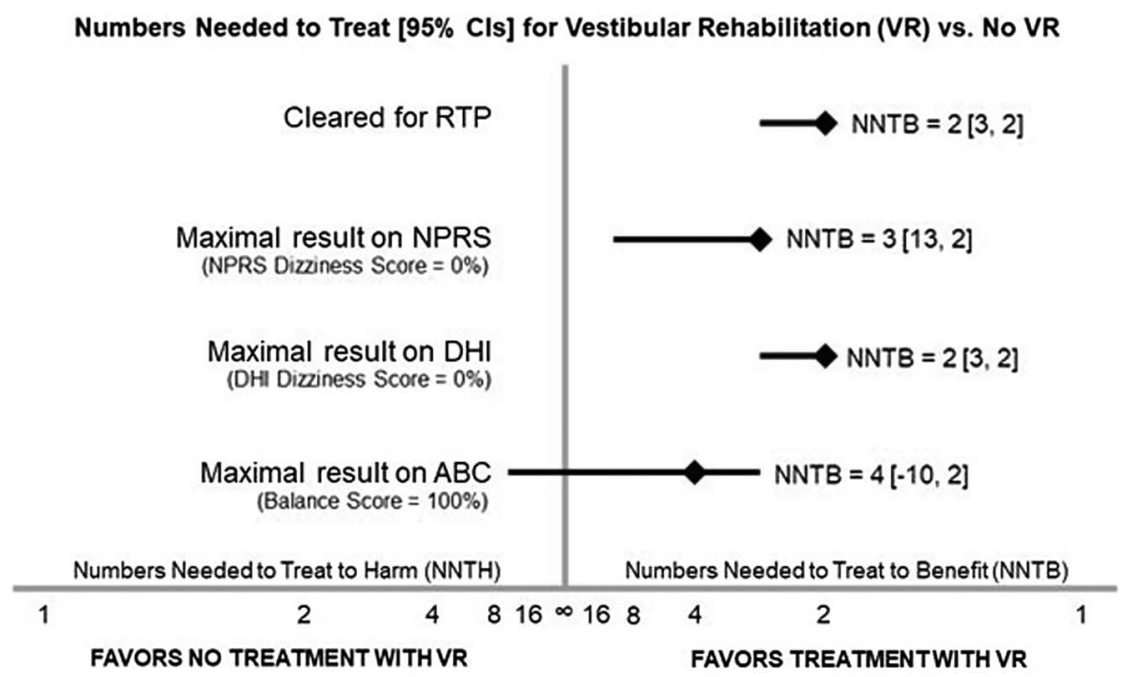

Figure 4 - A depiction of the numbers needed to treat [95\% CIs] for clearance to RTP, complete recovery of dizziness symptoms, and balance following VR compared to no VR. An estimated 3-13 individuals would need to be treated with VR to establish complete recovery in one. For balance scores, recovery with VR did appear beneficial, however the 95\% CI did encompass infinity. ABC = Assessment of Balance Confidence scale; $\mathrm{CI}=$ confidence interval; DHI = Dizziness Handicap Inventory scale; NNTB or NNTH = numbers needed to treat to benefit/harm; NPRS = Numeric Pain Rating Scale for Dizziness; RTP = return-to-play; VR = vestibular rehabilitation.

generally of low risk, ${ }^{5}$ but identifying the correct practitioner may be difficult for some athletes and ATs.

At this time, VRT is not required as part of athletic training professional preparation. ${ }^{21}$ The available evidence encompassed a wide variety of patients with concussion ${ }^{11-13}$ and only one study incorporated patients with sport-related concussion. ${ }^{13}$ However, the results of this CAT support the emerging recommendations for VRT in concussion management. ${ }^{7-9}$ It is recommended that athletic trainers actively identify concussion patients who are experiencing persistent vestibular symptoms through the use of subjective and objective assessments of dizziness and balance, and then to be prepared to refer concussed athletes with vestibular symptoms for appropriate care and treatment. Educating the patient on the importance of VRT is necessary to enhance compliance (agreeing to participate in a treatment protocol), adherence (sticking to that treatment protocol), and fidelity (participating in a treatment protocol faithfully) to the rehabilitation program, ${ }^{22-26}$ resulting in better treatment outcomes. ${ }^{27}$ Ultimately, future research may indicate a need for AT education to eventually include training in VRT. Until then, it is essential that we promote interprofessional practices that best support the health and well-being of our athletes who have sustained a concussion.

\section{CAT Kill Date: May 2021}

CATs have limited life and should be revisited approximately 2 years after publication (see https://doi.org/10.1123/ijatt.2018-0093).

\section{References}

1. McCrory P, Meeuwisse W, Dvořák J, et al. Consensus statement on concussion in sport-the 5th international conference on concussion in sport held in Berlin, October 2016. Br J Sports Med. 2017;51(11): 838-847. PubMed ID: 28446457 doi:10.1136/bjsports-2017-097699

2. Feddermann-Demont N, Echemendia RJ, Schneider KJ, et al. What domains of clinical function should be assessed after sport-related concussion? A systematic review. Br J Sports Med. 2017;51(11): 903-918. PubMed ID: 29098983. doi:10.1136/bjsports-2016-097403

3. McCrory P, Meeuwisse WH, Aubry M, et al. Consensus statement on concussion in sport: the 4th International Conference on Concussion in Sport, Zurich, November 2012. J Athl Train. 2013;48(4):554-575. PubMed ID: 23855364 doi:10.4085/1062-6050-48.4.05

4. McDonnell MN, Hillier SL. Vestibular rehabilitation for unilateral peripheral vestibular dysfunction. Cochrane Database Syst Rev. 2015;1:CD005397. PubMed ID: 25581507 doi:10.1002/14651858. CD005397.pub4

5. Quatman-Yates C, Cupp A, Gunsch C, Haley T, Vaculik S, Kujawa D. Physical rehabilitation interventions for post-mTBI symptoms lasting greater than 2 weeks: systematic review. Phys Ther. 2016;96(11):1753-1763. PubMed ID: 27197826. doi:10.2522/ptj. 20150557

6. Schneider KJ, Leddy JJ, Guskiewicz KM, et al. Rest and treatment/ rehabilitation following sport-related concussion: a systematic review. Br J Sports Med. 2017;51(12):930-934. PubMed ID: 28341726. doi:10.1136/bjsports-2016-097475

7. Broglio SP, Collins MW, Williams RM, Mucha A, Kontos AP. Current and emerging rehabilitation for concussion: a review of the evidence. Clin Sports Med. 2015;34(2):213-231. PubMed ID: 25818710. doi:10.1016/j.csm.2014.12.005

8. Marshall S, Bayley M, McCullagh S, et al. Updated clinical practice guidelines for concussion/mild traumatic brain injury and persistent symptoms. Brain Inj. 2015;29(6):688-700. PubMed ID: 25871303. doi:10.3109/02699052.2015.1004755

9. Murray DA, Meldrum D, Lennon O. Can vestibular rehabilitation exercises help patients with concussion? A systematic review of efficacy, prescription and progression patterns. Br J Sports Med. 2017;51(5):442-451. PubMed ID: 27655831. doi:10.1136/bjsports2016-096081

10. Wilkerson GB, Denegar CR. A growing consensus for change in interpretation of clinical research evidence. J Athl Train. 2018;53(3): 320-326. PubMed ID: 29624454. doi:10.4085/1062-6050-8-17

11. Alsalaheen BA, Mucha A, Morris LO, et al. Vestibular rehabilitation for dizziness and balance disorders after concussion. J Neurol Phys 
Ther. 2010;34(2):87-93. PubMed ID: 20588094. doi:10.1097/NPT. 0b013e3181dde568

12. Kleffelgaard I, Soberg HL, Bruusgaard KA, Tamber AL, Langhammer B. Vestibular rehabilitation after traumatic brain injury: Case series. J Phys Ther. 2016;96(6):839-849. doi:10.2522/ptj. 20150095

13. Schneider KJ, Meeuwisse WH, Nettel-Aguirre A, et al. Cervicovestibular rehabilitation in sport-related concussion: a randomised controlled trial. Br J Sports Med. 2014;48(17):1294-1298. PubMed ID: 24855132. doi:10.1136/bjsports-2013-093267

14. Kleffelgaard I, Soberg HL, Tamber AL, et al. The effects of vestibular rehabilitation on dizziness and balance problems in patients after traumatic brain injury: a randomized controlled trial. Clin Rehabil. 2019;33(1):74-84. PubMed ID: 30056743. doi:10.1177/ 0269215518791274

15. Kontos AP, Collins MW, Holland CL, et al. Preliminary evidence for improvement in symptoms, cognitive, vestibular, and oculomotor outcomes following targeted intervention with chronic mTBI patients. Mil Med. 2018;183(suppl 1):333-338. PubMed ID: 29635578. doi:10.1093/milmed/usx172

16. Moore BM, Adams JT, Barakatt E. Outcomes following a vestibular rehabilitation and aerobic training program to address persistent post-concussion symptoms. J Allied Health. 2016;45(4):e59-e68. PubMed ID: 27915363

17. McKeon PO, Medina McKeon JM. Outcomes assessment: demonstrating our predictive ability as a healthcare profession. Int J Athl Ther Train. 2016;21(4):1-4. doi:10.1123/ijatt.2016-0062

18. Jacobson GP, Newman CW. The development of the Dizziness Handicap Inventory. Arch Otolaryngol Head Neck Surg. 1990; 116(4):424-427. PubMed ID: 2317323. doi:10.1001/archotol.1990. 01870040046011
19. Goldsmith ES, Taylor BC, Greer N, et al. Focused evidence review: psychometric properties of patient-reported outcome measures for chronic musculoskeletal pain. J Gen Intern Med. 2018; 33(suppl 1):61-70. PubMed ID: 29633142. doi:10.1007/s11606-0184327-8

20. Leddy J, Baker JG, Haider MN, Hinds A, Willer B. A physiological approach to prolonged recovery from sport-related concussion. J Athl Train. 2017;52(3):299-308. PubMed ID: 28387557. doi:10.4085/ 1062-6050-51.11.08

21. Henderson J. The 2015 Athletic Trainer Practice Analysis Study. Omaha, NE: Board of Certification; 2015.

22. Niven A. Rehabilitation adherence in sport injury: sport physiotherapists' perceptions. J Sport Rehabil. 2007;16(2):93-110. PubMed ID: 17918697. doi:10.1123/jsr.16.2.93

23. Medina McKeon JM, McKeon PO. Patient evidence (AKA, boy, patients complicate our clinical practice). Int J Athl Ther Train. 2017; 22(6):1-4. doi:10.1123/ijatt.2017-0088

24. Howard JS, Sciascia A, Hoch JM. Using patient evidence to guide clinical care: consulting the other expert in the room. Int $J$ Athl Ther Train. 2018;23(2):53-56. doi:10.1123/ijatt.2018-0020

25. Fisher A, Scriber K, Matheny M, Alderman M, Bitting L. Enhancing athletic injury rehabilitation adherence. J Athl Train. 1993;28(4): 312-318. PubMed ID: 16558246

26. Gordon S, Milios D, Grove J. Psychological aspects of the recovery process from sport injury. Aust J Sci Med Sport. 1991;23(2): 53-60.

27. Brewer B, Cornelius A, Van Raalte J, et al. Age-related differences in predictors of adherence to rehabilitation after anterior cruciate ligament reconstruction. J Athl Train. 2003;38(2):158-162. PubMed ID: 12937528 